\title{
Role of mechanical and oral antibiotic bowel preparation in children with Hirschsprung's disease undergoing colostomy closure and pull-through
}

\author{
Yuanyuan Liang ${ }^{1,2 \#} \wedge$, Wenqiong $\mathrm{Xin}^{1,2 \#}$, Ling $\mathrm{Xi}^{1,2}$, Huan $\mathrm{Fu}^{1,2}$, Yang Yang ${ }^{1,2}$, Gang Yang ${ }^{1 \wedge}$, Xiaoling $\mathrm{Li}^{2}$ \\ ${ }^{1}$ Department of Pediatric Surgery, West China Hospital, Sichuan University, Chengdu, China; ${ }^{2}$ West China School of Nursing, Sichuan University, \\ Chengdu, China \\ Contributions: (I) Conception and design: Y Liang, W Xin; (II) Administrative support: G Yang, X Li; (III) Provision of study materials or patients: \\ Y Liang, L Xi; (IV) Collection and assembly of data: Y Liang, W Xin, H Fu, Y Yang; (V) Data analysis and interpretation: Y Liang, Y Yang; (VI) \\ Manuscript writing: All authors; (VII) Final approval of manuscript: All authors. \\ \#These authors contributed equally to this work. \\ Correspondence to: Gang Yang. Department of Pediatric Surgery, West China Hospital, Sichuan University, No. 37 Guoxue Xiang, Chengdu, \\ China. Email: emailtoyg@163.com; Xiaoling Li. West China School of Nursing, Sichuan University, No. 37 Guoxue Xiang, Chengdu, China. \\ Email: janifer818@163.com.
}

Background: Mechanical and oral antibiotic bowel preparation (MOABP) has been performed routinely before colorectal surgery in children, but the necessity was questioned recently. We evaluated the utility of MOABP in children with Hirschsprung's disease (HSCR) undergoing colostomy closure and pull-through.

Methods: The medical records of pediatric patients with HSCR who underwent colostomy closure and pull-through in a single center from January 2010 to January 2020 were reviewed. The use of MOABP was noted. The incidence of postoperative complications, duration of postoperative antibiotic therapy, total hospital cost and length-of-stay were compared between patients receiving MOABP and no bowel preparation (NBP).

Results: A total of 64 patients were included in the study: 33 received MOABP and 31 had NBP. The respective postoperative complications in the MOABP and NBP groups were: intra-abdominal infection (18.2\% vs. $29.0 \%$ ), wound infection ( $9.1 \%$ vs. 16.1\%), anastomotic leak (0 vs. 0), intestinal obstruction (6.1\% vs. 0$)$ and enterocolitis (3.03\% vs. $12.90 \%)$. The duration of antibiotic therapy was $4.91 \pm 4.21$ and $5.23 \pm 3.77$ days $(\mathrm{P}=0.75)$ and hospitalization was $18.21 \pm 7.26$ and $16.26 \pm 6.63$ days $(\mathrm{P}=0.27)$ respectively. The total hospital cost in the MOABP group $(4,720.14 \pm 1,858.89$ USD) was higher than in the NBP group $(3,749.06 \pm 2,009.97$ USD) $(\mathrm{P}=0.049)$.

Conclusions: We did not find any clear benefit of MOABP in children with HSCR before colostomy closure and pull-through. However, a multicenter randomized controlled trial is needed to more definitely determine the best preoperative approach for children with HSCR.

Keywords: Colostomy closure; complications; Hirschsprung's disease; mechanical bowel preparation

Submitted Aug 31, 2020. Accepted for publication Nov 06, 2020.

doi: $10.21037 /$ tp-20-306

View this article at: http://dx.doi.org/10.21037/tp-20-306

\footnotetext{
^ Yuanyuan Liang, ORCID: 0000-0002-8265-1645; Gang Yang, ORCID: 0000-0002-3016-2073.
} 


\section{Introduction}

Mechanical and oral antibiotic bowel preparation (MOABP) prior to colorectal surgery was thought necessary to remove feces from the large intestine, reduce the bacterial load in the lumen, and decrease surgical site infection (SSI) (1) or other postoperative complications $(2,3)$. However, doubts about the benefit of mechanical bowel preparation (MBP) in adults have been raised (4-11), and the randomized clinical trials, systematic reviews and meta-analyses of preoperative MBP in adults have concluded that it can be safely omitted (12-14). The benefit of MBP in children is still unclear because of the paucity of clinical evidencebased studies in general $(15,16)$. Several small studies done in pediatric elective colorectal surgical patients have found no differences in SSI incidence or other postoperative complications in pediatric patients who underwent MBP or not $(2,15,17-19)$. But the current trend in pediatric hospitals continues to favor use of MBP $(17,20)$. Breckler et al. (21) performed a survey in 2007 that revealed $96 \%$ of practicing pediatric surgeons used MBP in their practice. Especially for Hirschsprung's disease (HSCR), preoperative MBP is a widely accepted dogma, but MBP is a most unpleasant experience for both children and parents. It can cause dehydration, hypocalcemia, abdominal pain, distension, and fatigue $(4,5,22-27)$.

Hosseinpour et al. reported that they had found no clear benefit of MBP for young children with HSCR who were scheduled for Duhamel operation. However, more clinical trials are needed to validate the necessity of MBP in the pediatric population (28). So we conducted this retrospective study to explore whether omission of MOABP would increase the incidence of postoperative complications in infants and children with HSCR undergoing colostomy closure and pull-through. We present the following article in accordance with the STROBE reporting checklist (available at http://dx.doi.org/10.21037/tp-20-306).

\section{Methods}

\section{Study population}

After obtaining IRB approval (\#2020224), we performed a retrospective review of all available medical records of pediatric patients diagnosed with HSCR and who underwent colostomy closure and pull-through at West China Hospital, Sichuan University between January 2010 and January 2020. An attending pediatric surgeon and experienced research nurses reviewed each patient's medical record using the Health Information System database to maintain consistency of interpretation, accuracy and completeness of data. The inclusion criteria were: (I) aged 3 months to 14 years; and (II) diagnosed with HSCR and underwent colostomy closure and pull-through. The exclusion criteria were: (I) presence of severe underlying disease (e.g., heart, brain, and/or lung disease, and organ dysfunction), digestive tract malformation, and intellectual development disorder; (II) incomplete data; and (III) longsegment HSCR. All procedures performed in this study involving human participants were in accordance with the Declaration of Helsinki (as revised in 2013). Individual consent for this retrospective analysis was waived.

Patients were divided into two groups based on whether preoperative MOABP was performed or not. All operations were performed by two surgeons and the use of MOABP was at the discretion of the individual surgeon. Information was collected and compared for demographics, symptoms and pre-admission status (based on clinical assessment), and postoperative course, including any postoperative complications, duration of intravenous antibiotic therapy, combination of antibiotics, readmission for intravenous antibiotics, hospitalization, and total hospital cost. Complications included anastomotic leaks, intra-abdominal infection, wound infection, intestinal obstruction, enterocolitis and respiratory tract infection. The assignment of values of each variable was made by two of the authors (LY and $\mathrm{XW}$ ).

\section{Statistical analysis}

Data were processed using SPSS 22.0 software. Count data are expressed as cases, and a two-sample chi-square test or Fisher's exact test was used to compare the preoperative complications, the clinical manifestations, combined antibiotics and the postoperative complications of the two groups. Parametric data are expressed as mean \pm standard deviation, and a two-sample $t$-test was used to compare the ages, weights, duration of temporary colostomy, associated malformations, duration of antibiotic therapy, length and cost of hospital stay of the two groups. A P value $<0.05$ was considered significantly different.

\section{Results}

Based on the inclusion and exclusion criteria, a total of 64 patients comprised the study group (48 males, 16 females). The indication for colostomy included enterocolitis, severe 
Table 1 Patient demographics, symptoms and pre-admission status in the MOABP and NBP groups

\begin{tabular}{|c|c|c|c|c|}
\hline $\begin{array}{l}\text { Patient demographics, symptoms and } \\
\text { pre-admission status }\end{array}$ & $\operatorname{MOABP}(n=33)$ & $\operatorname{NBP}(n=31)$ & $P$ value & OR $(95 \% \mathrm{Cl})$ \\
\hline \multicolumn{5}{|l|}{ Patient demographics } \\
\hline Age (months) & $7.96 \pm 3.98$ & $8.16 \pm 5.22$ & 0.88 & NA \\
\hline \multicolumn{5}{|l|}{ Symptoms and pre-admission status } \\
\hline Preoperative constipation & $0(0)$ & $0(0)$ & NA & NA \\
\hline Preoperative abdominal distension & $3(9.1 \%)$ & $0(0)$ & 0.24 & $0.49(0.38-0.64)$ \\
\hline Preoperative colostomy complications & $7(21.2 \%)$ & $3(9.1 \%)$ & 0.31 & $2.51(0.59-10.76)$ \\
\hline
\end{tabular}

$\mathrm{Cl}$, confidence interval; MOABP, mechanical and oral antibiotic bowel preparation; NBP, no bowel preparation; OR, odds ratio; NA, Not applicable (retrospective study).

abdominal distension, intestinal perforation in a newborn and overdilated proximal colon. The diagnosis of HSCR was confirmed by rectal biopsy in all patients. In this study 33 patients underwent MOABP, and 31 patients underwent no bowel preparation (NBP). All patients received perioperative intravenous antibiotics, including cefoxitin, ampicillin, cefmetazole, clindamycin, monocyclic-lactamase or imipenem cilastatin. Seven patients in each group were given a combination of antibiotics. In the MOABP group, gentamicin and metronidazole were routinely taken orally for 3 days before the operation, and intestinal irrigation with normal saline was performed once daily, including on the day before the operation. The patients in each group were similar in sex, age, weight, duration of temporary colostomy, associated malformations, occurrence of preoperative constipation, abdominal distension, vomiting, enterocolitis, water and electrolyte disorder, and colostomy complications. Demographic data, symptoms and preadmission status are presented in Table 1 .

There were no statistically significant differences in duration of antibiotic therapy, combined antibiotics, or length of hospital stay between the two groups. But the cost of hospital stay in the MOABP group $(4,720.14 \pm 1,858.89$ USD) was higher than in the NBP group $(3,749.06 \pm 2,009.97$ USD) $(\mathrm{P}=0.04)$. Postoperative complications were uncommon in both groups of patients
(Table 2). In the MOABP group there were two cases of intestinal obstruction, which were managed with bowel rest, antibiotics, and total parenteral nutrition. Other complications were managed with antibiotics.

\section{Discussion}

Because MOABP before colorectal surgery was believed to help in relieving bloating and reducing the incidence of complications such as anastomotic dehiscence, wound infection and sepsis, it was considered the standard of care by colorectal surgeons for almost half a century. But in the past decades the value of MOABP has been questioned. In our study, omitting MOABP did not increase the occurrence of postoperative intra-abdominal infections, wound infections, anastomotic leak, intestinal obstruction or enterocolitis. That result was in accordance with a recent randomized control trial in adults, which compared MOABP with NBP in patients who were scheduled for colon resection and suggested that MOABP did not reduce the occurrence of SSIs or overall morbidity after colonic surgery (29). A meta-analysis including a total of 5,805 participants also did not show any statistically significant evidence that patients benefit from either MBP or the use of rectal enemas. Accordingly, it is suggested that bowel cleansing can be safely omitted in colonic surgery without 
Table 2 Postoperative treatment and complications in the MOABP and NBP groups

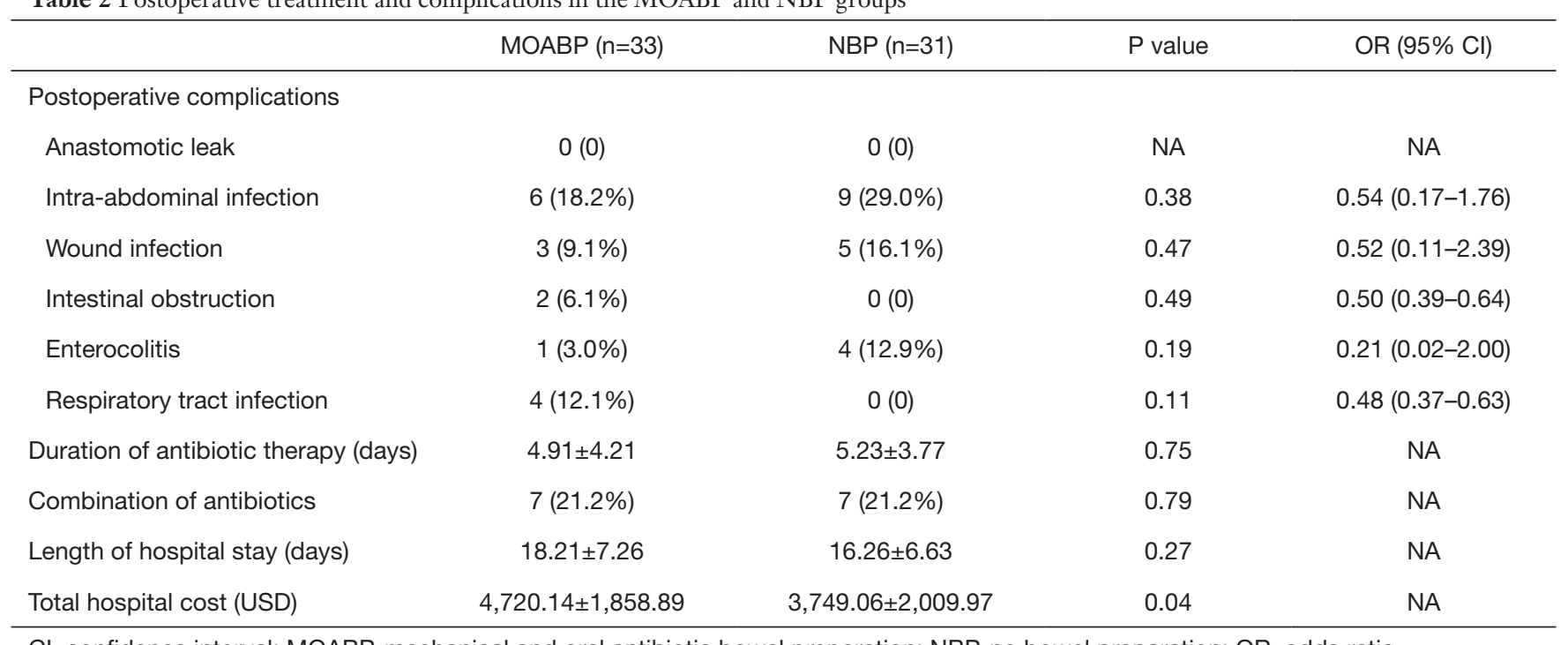

$\mathrm{Cl}$, confidence interval; MOABP, mechanical and oral antibiotic bowel preparation; NBP, no bowel preparation; OR, odds ratio.

inducing a higher complication rate.

However, results from other studies suggest that the use of MOABP is associated with more favorable outcomes (30-34). The largest study involves three large retrospective cohort studies of the American College of Surgeons National Surgical Quality Improvement Program (ACSNSQIP), targeting colectomy data $(32,35,36)$. A total of 45,724 elective colectomies with anastomosis were performed from 2012 to 2015 . The combination of MBP and oral antibiotics was associated with lower rates of SSIs, anastomotic leaks and 30-day mortality. However, all evidence to date is based on studies in adults. Pediatric patients are so different from adults in the diagnosis, surgical methods, underlying diseases, immunity status and gut flora, so the results from adults cannot simply be extrapolated to pediatric patients. Furthermore, the MBP process has potential adverse effects and is associated with patient dissatisfaction rates (37). It is also a lot of work for nurses. The same conclusion has been reached in other studies of pediatric surgery $(18,38,39)$, and some studies even suggest that MBP could liquefy solid stools, increasing the chances of intraoperative spillage and contamination $(40,41)$. A growing body of literature data suggests that the microbiota has colon resistant effects (42-44), and theoretically rectal washout and MBP can change the composition of the colonic microbiota and affect bowel function. In the current study, the two patients $(6.06 \%)$ with postoperative intestinal obstructions were in the MOABP group, and no cases of intestinal obstruction occurred in the
NBP group; although this was not statistically significant, which may be related to the small sample size. A survey by the American Pediatric Surgical Association on bowel preparation practices by pediatric surgeons revealed that $31 \%$ used MBP alone, 27\% used diet modification only, $20 \%$ used MOABP, and $12 \%$ did not use any preoperative prep (20). This identified a trend towards no preoperative bowel regimen as the strategy of choice.

In this study, hospitalization of MOABP group had a higher cost than the NBP group, although there was no significant difference between the two groups in the length of hospital stay because the MOABP patients were not admitted early to hospital for the bowel preparation, part of which was completed before admission.

\section{Study limitations}

First, this was a retrospective study and patients were not randomly assigned to the MOABP or NBP groups. The decision for MOABP was based on surgeon preference and resulted in selected bias. Second, our results must be interpreted with caution, given the relatively small number of study participants. Finally, the recording may be incomplete that created potential for a significant confounding factor in this study. To overcome confounding factors and other factors in retrospective studies such as comparison of individual surgeon's practices, a large, prospective randomized clinical trial is needed to validate our findings. 
Our study indicated that the quality of evidence for the use of MOABP before HSCR colostomy closure and pullthrough is low. On the basis of existing evidence, MBP seems not to 'decrease the incidence of anastomotic leakage, intra-abdominal infection, or wound infection' compared with NBP and may therefore be safely omitted prior to HSCR colostomy closure surgery in children.

\section{Acknowledgments}

Funding: West China Nursing Discipline Development Special Fund Project, Sichuan University (No. HXHL19046).

\section{Footnote}

Reporting Checklist: The authors have completed the STROBE reporting checklist. Available at http://dx.doi. org/10.21037/tp-20-306

Data Sharing Statement: Available at http://dx.doi. org/10.21037/tp-20-306

Conflicts of Interest: All authors have completed the ICMJE uniform disclosure form (available at http://dx.doi. org/10.21037/tp-20-306). The authors have no conflicts of interest to declare.

Ethical Statement: The authors are accountable for all aspects of the work in ensuring that questions related to the accuracy or integrity of any part of the work are appropriately investigated and resolved. The study was approved by the Ethics Committee of The West China Hospital, Sichuan University (\#2020224). All procedures performed in this study involving human participants were in accordance with the Declaration of Helsinki (as revised in 2013). Individual consent for this retrospective analysis was waived.

Disclaimer: The views expressed in the submitted article are the authors' own.

Open Access Statement: This is an Open Access article distributed in accordance with the Creative Commons Attribution-NonCommercial-NoDerivs 4.0 International License (CC BY-NC-ND 4.0), which permits the noncommercial replication and distribution of the article with the strict proviso that no changes or edits are made and the original work is properly cited (including links to both the formal publication through the relevant DOI and the license). See: https://creativecommons.org/licenses/by-nc-nd/4.0/.

\section{References}

1. Saha AK, Chowdhury F, Jha AK, et al. Mechanical bowel preparation versus no preparation before colorectal surgery: A randomized prospective trial in a tertiary care institute. J Nat Sci Biol Med2014;5:421-4.

2. Janssen Lok M, Miyake H, O'Connell JS, et al. The value of mechanical bowel preparation prior to pediatric colorectal surgery: a systematic review and meta-analysis. Pediatr Surg Int 2018;34:1305-20.

3. Frizelle FA, Colls BM. Hyponatremia and seizures after bowel preparation: report of three cases. Dis Colon Rectum 2005;48:393-6.

4. Abis GSA, Stockmann H, Bonjer HJ, et al. Randomized clinical trial of selective decontamination of the digestive tract in elective colorectal cancer surgery (SELECT trial). Br J Surg 2019;106:355-63.

5. Zmora O, Mahajna A, Bar-Zakai B, et al. Colon and rectal surgery without mechanical bowel preparation: a randomized prospective trial. Ann Surg 2003;237:363-7.

6. Espin Basany E, Solís-Peña A, Pellino G, et al. Preoperative oral antibiotics and surgical-site infections in colon surgery (ORALEV): a multicentre, singleblind, pragmatic, randomised controlled trial. Lancet Gastroenterol Hepatol 2020;5:729-38.

7. Bucher P, Mermillod B, Morel P, et al. Does mechanical bowel preparation have a role in preventing postoperative complications in elective colorectal surgery? Swiss Med Wkly 2004;134:69-74.

8. Zorbas KA, Yu D, Choudhry A, et al. Preoperative bowel preparation does not favor the management of colorectal anastomotic leak. World J Gastrointest Surg 2019;11:218-28.

9. van Geldere D, Fa-Si-Oen P, Noach LA, et al. Complications after colorectal surgery without mechanical bowel preparation. J Am Coll Surg 2002;194:40-7.

10. Treppiedi E, La Mendola R, Rosso E. 2019. Bowel preparation in elective colorectal surgery: back to the future? Updates Surg 2019;71:595-6.

11. Leenen JPL, Hentzen J, Ockhuijsen HDL. Effectiveness of mechanical bowel preparation versus no preparation on anastomotic leakage in colorectal surgery: a systematic review and meta-analysis. Updates Surg 2019;71:227-36.

12. Rollins KE, Javanmard-Emamghissi H, Lobo DN. 
Impact of mechanical bowel preparation in elective colorectal surgery: A meta-analysis. World J Gastroenterol 2018;24:519-36.

13. Fry DE. Colon preparation and surgical site infection. Am J Surg 2011;202:225-32.

14. Contant CM, Hop WC, van't Sant HP, et al. Mechanical bowel preparation for elective colorectal surgery: a multicentre randomised trial. Lancet 2007;370:2112-7.

15. Ares GJ, Helenowski I, Hunter CJ, et al. Effect of preadmission bowel preparation on outcomes of elective colorectal procedures in young children. J Pediatr Surg 2018;53:704-7.

16. Zwart K, Van Ginkel DJ, Hulsker CCC, et al. Does Mechanical Bowel Preparation Reduce the Risk of Developing Infectious Complications in Pediatric Colorectal Surgery? A Systematic Review and MetaAnalysis. J Pediatr 2018;203:288-293.e1.

17. Pennington EC, Feng C, St Peter SD, et al. Use of mechanical bowel preparation and oral antibiotics for elective colorectal procedures in children: is current practice evidence-based? J Pediatr Surg 2014;49:1030-5.

18. Aldrink JH, McManaway C, Wang W, et al. Mechanical bowel preparation for children undergoing elective colorectal surgery. J Pediatr Gastroenterol Nutr 2015;60:503-7.

19. Leys CM, Austin MT, Pietsch JB, et al. Elective intestinal operations in infants and children without mechanical bowel preparation: a pilot study. J Pediatr Surg 2005;40:978-81; discussion 982.

20. Feng C, Sidhwa F, Anandalwar S, et al. Variation in bowel preparation among pediatric surgeons for elective colorectal surgery: A problem of equipoise or a knowledge gap of the available clinical evidence? J Pediatr Surg 2015;50:967-71.

21. Breckler FD, Fuchs JR, Rescorla FJ. Survey of pediatric surgeons on current practices of bowel preparation for elective colorectal surgery in children. Am J Surg 2007;193:315-8.

22. Holte K, Nielsen KG, Madsen JL, et al. Physiologic effects of bowel preparation. Dis Colon Rectum 2004;47:1397-402.

23. Lieberman DA, Ghormley J, Flora K. Effect of oral sodium phosphate colon preparation on serum electrolytes in patients with normal serum creatinine. Gastrointest Endosc 1996;43:467-9.

24. Ram E, Sherman Y, Weil R, et al. Is mechanical bowel preparation mandatory for elective colon surgery? A prospective randomized study. Arch Surg 2005;140:285-8.
25. Zmora O, Mahajna A, Bar-Zakai B, et al. Is mechanical bowel preparation mandatory for left-sided colonic anastomosis? Results of a prospective randomized trial. Tech Coloproctol 2006;10:131-5.

26. Sinha SK, Kanojia RP, Rawat JD, et al. Comparison of three solutions for total gut irrigation in pediatric patients. Pediatr Surg Int 2007;23:581-4.

27. Vejzovic V, Wennick A, Idvall E, et al. A private affair: children's experiences prior to colonoscopy. J Clin Nurs 2015;24:1038-47.

28. Hosseinpour M, Ahmadi B, Etezazian S. Mechanical Bowel Preparation versus No Preparation in Duhamel Procedure in Children with Hirschsprung's Disease. Eur J Pediatr Surg 2020;30:201-4.

29. Koskenvuo L, Lehtonen T, Koskensalo S, et al. Mechanical and oral antibiotic bowel preparation versus no bowel preparation for elective colectomy (MOBILE): a multicentre, randomised, parallel, single-blinded trial. Lancet 2019;394:840-8.

30. Morris MS, Graham LA, Chu DI, et al. Oral Antibiotic Bowel Preparation Significantly Reduces Surgical Site Infection Rates and Readmission Rates in Elective Colorectal Surgery. Ann Surg 2015;261:1034-40.

31. Zhang X, Li T, Li Y, et al. Protective effect of intraoperative re-dose of prophylactic antibiotics on surgical site infection in diabetic patients: a retrospective cohort study. Ann Transl Med 2019;7:96.

32. Kiran RP, Murray AC, Chiuzan C, et al. Combined preoperative mechanical bowel preparation with oral antibiotics significantly reduces surgical site infection, anastomotic leak, and ileus after colorectal surgery. Ann Surg 2015;262:416-25.

33. Nelson RL, Gladman E, Barbateskovic M. Antimicrobial prophylaxis for colorectal surgery. Cochrane Database Syst Rev 2014;2014;CD001181.

34. Kelay A, Tullie L, Stanton M. Surgery and paediatric inflammatory bowel disease. Transl Pediatr 2019;8:436-48.

35. Midura EF, Jung AD, Hanseman DJ, et al. Combination oral and mechanical bowel preparations decreases complications in both right and left colectomy. Surgery 2018;163:528-34.

36. Klinger AL, Green H, Monlezun DJ, et al. The Role of Bowel Preparation in Colorectal Surgery: Results of the 2012-2015 ACS-NSQIP Data. Ann Surg 2019;269:671-7.

37. Jung B, Matthiessen P, Smedh K, et al. Mechanical bowel preparation does not affect the intramucosal bacterial colony count. Int J Colorectal Dis 2010;25:439-42.

38. Slim K, Vicaut E, Launay-Savary MV, et al. Updated 
systematic review and meta-analysis of randomized clinical trials on the role of mechanical bowel preparation before colorectal surgery. Ann Surg 2009;249:203-9.

39. Slim K, Vicaut E, Panis Y, et al. Meta-analysis of randomized clinical trials of colorectal surgery with or without mechanical bowel preparation. Br J Surg 2004;91:1125-30.

40. Serrurier K, Liu J, Breckler F, et al. A multicenter evaluation of the role of mechanical bowel preparation in pediatric colostomy takedown. J Pediatr Surg 2012;47:190-3.

41. Mahajna A, Krausz M, Rosin D, et al. Bowel preparation

Cite this article as: Liang $\mathrm{Y}, \mathrm{Xin} \mathrm{W}, \mathrm{Xi} \mathrm{L}, \mathrm{Fu} \mathrm{H}$, Yang Y, Yang G, Li X. Role of mechanical and oral antibiotic bowel preparation in children with Hirschsprung's disease undergoing colostomy closure and pull-through. Transl Pediatr 2021;10(1):153-159. doi: 10.21037/tp-20-306 is associated with spillage of bowel contents in colorectal surgery. Dis Colon Rectum 2005;48:1626-31.

42. Jandhyala SM, Talukdar R, Subramanyam C, et al. Role of the normal gut microbiota. World J Gastroenterol 2015;21:8787-803.

43. Tuddenham S, Sears CL. The intestinal microbiome and health. Curr Opin Infect Dis 2015;28:464-70.

44. Valdes AM, Walter J, Segal E, et al. Role of the gut microbiota in nutrition and health. BMJ 2018;361:k2179.

(English Language Editor: K. Brown) 